\title{
The prefrontal cortex of the primate: A synopsis
}

\author{
JOAQUÍN M. FUSTER \\ University of California, Los Angeles, California
}

\begin{abstract}
The prefrontal cortex is one of the latest regions of the neocortex to develop, in both phylogeny and ontogeny. In the primate, the prefrontal cortex is anatomically divided into three major sectors: medial, orbital (or inferior), and dorsolateral. The dorsolateral sector is the association cortex of the convexity of the frontal lobe. Phylogenetically and ontogenetically, this part of the prefrontal cortex is the one to develop last and most. It is the neural substrate of the higher cognitive functions that reach their maximum development in the human brain. The most general and distinctive function of the dorsolateral prefrontal cortex is the temporal organization of goal-directed actions. In the human, this role extends to the domains of speech and reasoning. Two temporally symmetrical and mutually complementary cognitive functions - one retrospective and the other prospective- support that general prefrontal function of temporal organization: (1) active short-term memory, also called working memory; (2) prospective or preparatory set. The dorsolateral prefrontal cortex interacts with other cortical and subcortical structures in those two time-bridging functions at the basis of the temporal organization of behavior.
\end{abstract}

This article presents in summary form the most salient empirical facts known to date on the structure and functions of the prefrontal cortex of the human and nonhuman primate. The available evidence is here organized around a core theory of the cognitive functions of this cortex that has developed for the most part during the past quarter century, although it has long historical roots in human and simian neuropsychology. Briefly, the theory states that the cardinal function of the prefrontal cortex is the organization of goal-directed behavior in the temporal domain. This cardinal function of ensuring the orderly and timely execution of sequential acts extends, in the human, to speech and reasoning. Different but interconnected subdivisions of the prefrontal cortex support the temporal organization of different aspects of behavioral and cognitive activity. Elsewhere (Fuster, 1997) I review in greater detail both the theory and the facts that support it.

\section{Morphology}

Phylogenetically (Brodmann, 1912; Jerison, 1994) and ontogenetically (Conel, 1939-1963; Huttenlocher, 1979), the prefrontal cortex develops more slowly and further than most other parts of the brain (Figure 1). It is one of the last domains of the neocortex to develop. The human prefrontal cortex does not reach full structural maturation until young adulthood. In the adult, it constitutes nearly one third of the entirety of the neocortex.

The prefrontal cortex of the primate is divided into three major parts (Figure 2): (1) dorsolateral - that is,

Correspondence should be addressed to J. M. Fuster, UCLA Neuropsychiatric Institute, 760 Westwood Plaza, Los Angeles, CA 90024 (e-mail: joaquinf@ucla.edu). the cortex of the dorsal and lateral convexity of the anterior part of the frontal lobe (Brodmann's cytoarchitectonic area 46 , and lateral parts of areas $8,9,10$, and 11 ); (2) medial and cingulate, nearly flat and facing the medial surface of the contralateral frontal pole (areas 12, 24, and 32, and medial parts of $8,9,10$, and 11); and (3) inferior or orbital, slightly concave and directly over the orbit (areas 13,47 , and inferior parts of 10,11 , and 13). Of the three, the dorsolateral region develops last and most. It is bordered posteriorly by the premotor cortex (area 6); the medial and orbital prefrontal cortices (often considered together under the designation of "orbitomedial prefrontal cortex") are anterior and contiguous to the corpus callosum and limbic structures (piriform cortex and amygdala, cingulate cortex, septum, and hypothalamus). Cytoarchitecturally, the posterior orbitomedial cortex is transitional, with features of both limbic cortex and neocortex. It is sometimes considered "paralimbic prefrontal cortex."

\section{Functional Connectivity}

All the functions of the prefrontal cortex may be considered components of a general role of the frontal cortex in the representation and execution of actions in and by the organism. The cortex of the frontal lobe, in its entirety, can be viewed as "motor cortex" in the broadest sense of the term. That vast cortical territory represents and orchestrates actions in all aspects of adaptation of the organism to its internal and external environment. To coordinate such a wide range of activities, the prefrontal cortex in particular is connected with a wide variety of cerebral structures. The prefrontal cortex is one of the best connected of all neocortical regions, at least in terms of the number of cerebral structures with which it is connected. 

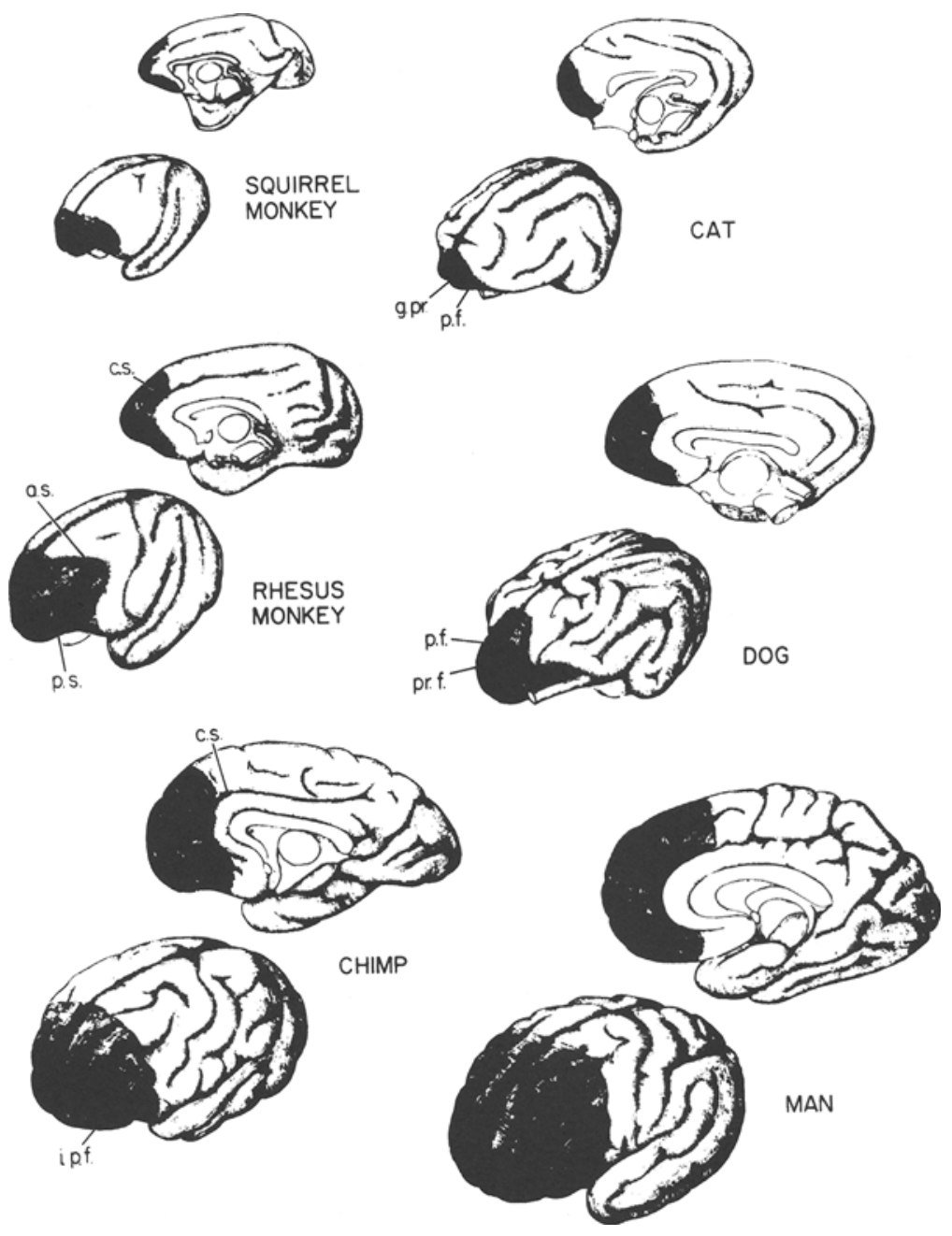

Figure 1. The prefrontal cortex (dark shading) in six animal species.

Every one of the three major prefrontal regions has its own connections - mainly reciprocal and organized topologically - with the other two regions. In addition, all three are connected with other structures of the brain, both cortical and subcortical. The most important, for their quantity and functional significance, are the connections with the mediodorsal thalamus (Akert, 1964; Van Buren \& Borke, 1972); with several limbic structures, notably the hippocampus and the amygdala (Goldman-Rakic, Selemon, \& Schwartz, 1984; Morecraft, Geula, \& Mesulam, 1992; Porrino, Crane, \& Goldman-Rakic, 1981); with the basal ganglia (Haber, Kunishio, Mizobuchi, \& Lynd-Balta, 1995; Selemon \& Goldman-Rakic, 1985); and with other regions of the cerebral cortex (Pandya \& Yeterian, 1985).

The orbital and medial prefrontal areas are especially well connected with the medial and anterior nuclei of the thalamus, the prepiriform cortex, the hippocampus, the amygdala, and the hypothalamus. This connectivity with the thalamus and limbic structures supports three cate- gories of prefrontal function: (1) processing of information on taste and olfaction; (2) regulation of the "internal milieu" of the organism-through the hypothalamus and the viscera -in relation to drive, motivation, and affective states; and (3) control of instinctual, emotional, and social behavior.

The cortex of the dorsolateral prefrontal convexity is profusely connected with other frontal areas homolaterally and - through the corpus callosum - contralaterally, with the hippocampus, and with the temporal and parietal cortex. All this connectivity serves cognition. It supports the associative functions of executive memory and the temporal organization of behavior. Further, the dorsolateral prefrontal areas send important efferent fibers to the basal ganglia, including the caudate nucleus, and the cerebellum. This connectivity serves the motor output functions of the prefrontal cortex through structures that are involved in skeletal and ocular motilities.

Two large categories of connections are of special interest as they relate to the cognitive functions of the pre- 


\section{DORSOLATERAL}
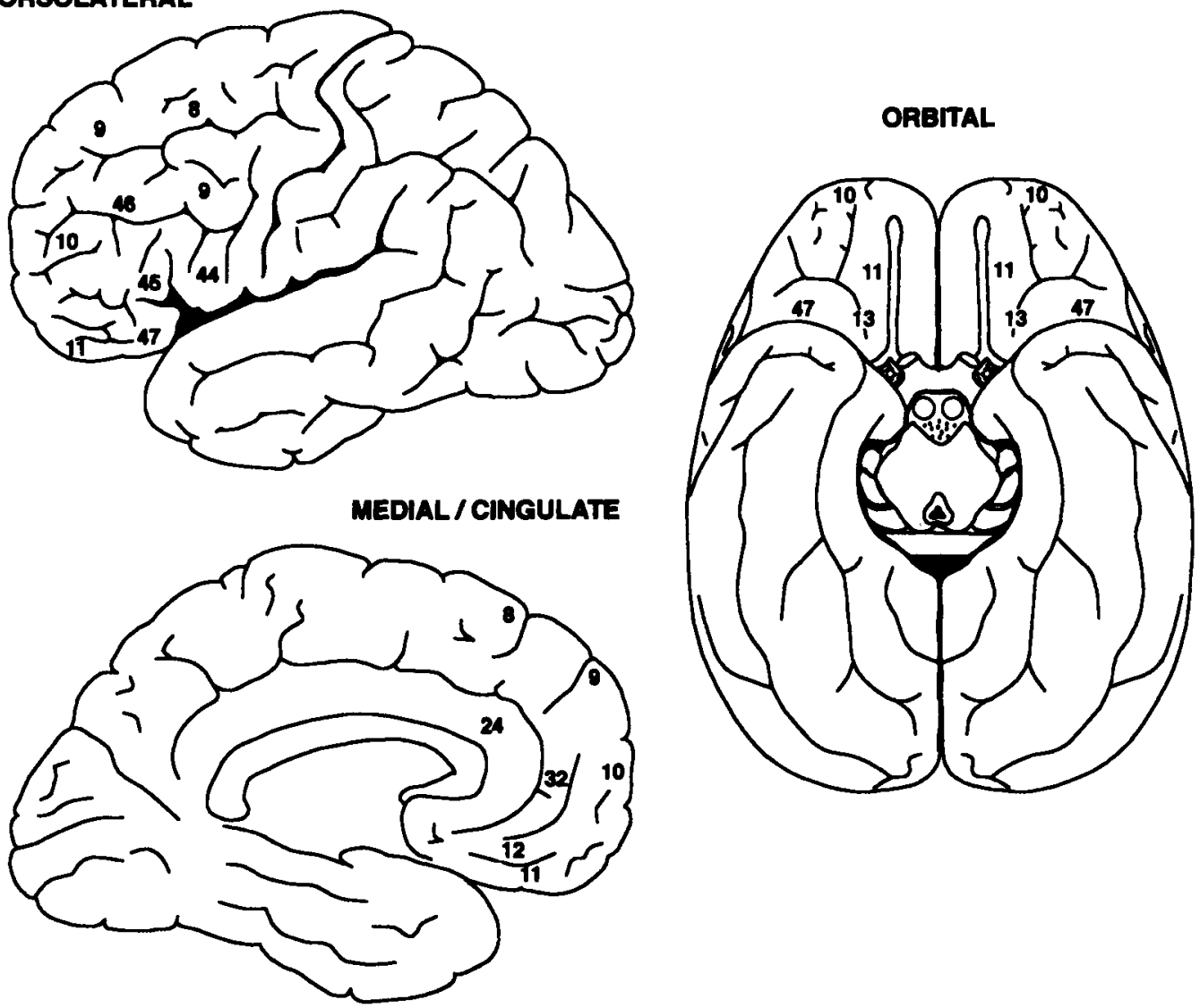

Figure 2. The three major regions of the prefrontal cortex in the human, with cytoarchitectonic areas numerated according to Brodmann's map.

frontal cortex. One is the connectivity with the hippocampus, reciprocal with the dorsolateral as well as the ventromedial prefrontal cortex, though apparently more abundant with the latter (Amaral, 1987; Morecraft et al., 1992). The functions of hippocampus-prefrontal connections have not been established with certainty. It is reasonable to suppose, however, that they are involved in the formation and retrieval of memory, especially motor (executive) memory.

The other order of connections implicated in cognition are those that link the prefrontal cortex with posterior (postrolandic) regions of the neocortex (Cavada \& Goldman-Rakic, 1989; Jones \& Powell, 1970; Pandya \& Yeterian, 1985). Most probably, these connections mediate not only the establishment and retrieval of executive memory but the activation of such memory for the short term. It appears that loops of excitatory reverberation between the dorsolateral prefrontal cortex and posterior cortical areas are probably essential in the maintenance of working memory (Fuster, Bauer, \& Jervey, 1985; Zipser, Kehoe, Littlewort, \& Fuster, 1993).

\section{Behavioral Neurophysiology}

Orbital and medial prefrontal areas exert inhibitory control of internal drives. Monkeys and humans with lesions of the orbitomedial cortex often manifest disinhibition of eating, sex, and aggression, with related alterations of social and emotional behavior. Further, insofar as impulse control is required for the concentration of attention and for new and complex goal-directed behavior, the orbitomedial prefrontal cortex serves the temporal organization of such behavior. This cortex helps keep in check internal impulses that may interfere with it. The inhibitory control function of the prefrontal cortex is probably mediated, in large part, by efferent connections to the basal ganglia (Selemon \& Goldman-Rakic, 1985) and the hypothalamus (Jacobson, Butters, \& Tovsky, 1978).

The dorsolateral prefrontal cortex, on the other hand, provides the cognitive support to the temporal organization of behavior, as well as to language and reasoning. That cognitive support consists of two basic aspects of neural function: representation and execution. The representations in the dorsolateral prefrontal cortex fall un- 
der the general category of motor or executive memory. This kind of memory probably consists of widely distributed neuronal networks representing schemes of temporally organized action in the form of general plans, procedures, or programs of behavior. Included are complex schemes of behavior that have been recently acquired. The qualifications of novelty and complexity are important, because, after it has been firmly consolidated, automatic or routine behavior appears represented and enacted by neural structures at lower stages of the motor hierarchy (e.g., motor and premotor cortices, basal ganglia).

As noted above, the connections of the prefrontal cortex with the hippocampus are probably involved in the acquisition of executive memory. The networks of executive memory seem to be organized hierarchically in the cortex of the frontal lobe. In the primary motor cortex, the lowest stage of that organization, are represented the most elementary motor memories, defined by specific movement and muscle group (this is what has been considered innate or "phyletic" motor memory); above, in the premotor cortex, are motor representations defined by goal and trajectory of movement; at the top, in the dorsolateral prefrontal cortex, are the representations of schemes and sequences of goal-directed behavior. In a manner still largely unknown, the dorsolateral prefrontal region represents the broad schemes of recent and prospective action. Further, it is critically involved in the enactment of those schemes. In the human, the inability to formulate and carry out plans is a consistent component of the clinical syndrome that follows upon large lesions of the dorsolateral prefrontal cortex.

\section{Temporal Organization}

In the primate, the principal function of the dorsolateral prefrontal cortex is the temporal organization of behavior and -in the human primate - of language and reasoning. Considerable evidence from monkeys and human subjects indicates that this cortex ensures the timely execution of all new and complex goal-directed sequences in those three activity domains (Fuster, 1997; Luria, 1966). That execution requires a preexisting neural representation of broad schemes or mappings of action, as postulated in the previous section. The enactment of each sequence is a complex process of temporal integration involving many cortical and subcortical structures of the brain. That process is orchestrated by the dorsolateral prefrontal cortex. This cortex is then ultimately responsible for the formation of new temporal gestalts or "melodies" of action toward the attainment of cognitive and biological goals.

In the past 25 years, physiological evidence has been obtained for two temporally integrative functions of the dorsolateral prefrontal cortex that support the temporal integration of behavior. One is active short-term mem$o r y$, the memory for recent information that needs to be retained for performance of an action in the near future. The other is prospective set, the preparation of the motor apparatus and the sensorium for that anticipated action.
The two functions are temporally symmetrical, the first retrospective and the second prospective. They are mutually complementary and help the organism to mediate cross-temporal contingencies of behavior-in other words, to bridge time between sensory inputs and consequent acts. Both are based on the physiological cooperation of the dorsolateral prefrontal cortex with associative regions of the posterior cortex.

Active short-term memory. The notion of short-term memory usually refers to the temporary retention of information before it becomes established in long-term memory. Cognitive psychology, however, has introduced a different concept for what has been construed as a different kind of short-term memory: the temporary retention of information for the execution of an act in the near term, as often required in complex behavior and for the solution of problems. This kind of short-term memory has been named "working memory" (Baddeley, 1986). The neurobiological evidence is now mounting that both kinds of short-term memory use the same cortical neurons and networks, and that working memory is essentially the sustained activation of a cortical network representing information to be used prospectively in the near term (Fuster, 1995). That information may be recently acquired anew, or it may be an item of long-term memory reactivated for the short term. Insofar as that memory, old or new, contributes to the formation of a new temporal structure of behavior, the dorsolateral prefrontal cortex and its networks become involved.

The evidence for the participation of the dorsolateral prefrontal cortex in active short-term memory derives mainly from the use of two basic methodologies: one is the inactivation of this cortex and the study of its effects on performance of short-term memory tasks (delay tasks); the other is the microelectrode recording of single-cell potentials in animals performing those tasks. In such tasks - for example, delayed response, delayed matching - the animal is presented on repeated trials with a piece of information that varies from trial to trial and is obliged to retain it for a few seconds in order to execute an appropriate motor act in response to it. Because the memorandum changes at random between trials and is part of a repertoire acquired by the animal on learning the task, every trial is essentially a new structure of behavior formed by the use of old memory, a new play in an old game. Animals with damaged dorsolateral prefrontal cortex cannot preform the task correctly, failing especially at long delay intervals (memorization periods). They are incapable of retaining the memorandum in working memory. This does not mean that the animals cannot retain new cues or discriminanda for long periods (e.g., $24 \mathrm{~h}$ or longer). They can (Bachevalier \& Mishkin, 1986), the reason probably being that those stimuli can gain access to long-term memory, despite the prefrontal lesion, when there are no other competing stimuli that induce ambiguities or uncertainties in the task at hand (as is the case in delay tasks). The inhibition of such competitive stimuli is usually compromised by prefrontal dam- 
age, especially if the damage is orbitomedial. In any case, whether the prefrontally damaged animal is or is not prevented from acquiring a new executive memory depends on the complexity of that memory and of the sensory stimuli that guide it.

In the normal monkey performing a delay task, neurons of dorsolateral prefrontal cortex fire in sustained-often stimulus-specific-manner during the delay, the memory period of every trial (Funahashi, Bruce, \& GoldmanRakic, 1989; Fuster, 1973; Niki, 1974). That sustained discharge, especially when it is stimulus specific, reflects the memorization of the cue. It is a neurophysiological correlate of the activation of the cortical memory network that represents the cue. In some cells the sustained activation during the delay reflects, instead, the events in preparation - namely, the motor response and the anticipated sensory stimuli immediately releasing that response. These cells represent in active form the prospective components of the structure of the trial (see the next section).

In the past 10 years, advances in neuroimaging, notably the advent of fMRI and the greater spatial as well as temporal resolution of brain images that they afford, have led to the visualization of the role of the human prefrontal cortex in active short-term memory (Cohen et al., 1994; Jonides et al., 1993; McCarthy et al., 1994; Swartz et al., 1995). The new methodology, however, still has serious handicaps to overcome (e.g., intersubject variability, arbitrariness of threshold setting, inability to expose neural inhibition, ignorance of the neural-hemodynamic coupling function). Before these handicaps have been overcome, neuroimaging has produced unmistakable correlates of prefrontal activation with active short-term (working) memory. The topographic definition of such correlates, however, is still limited and subject to continuing revision.

The critical attribute of active short-term memory and the role that the prefrontal cortex plays in it is the teleological character of that memory. That memory has been mobilized for the structuring of future action to attain a goal. Microelectrode studies suggest that different areas of the dorsolateral prefrontal cortex are specialized in retaining different types of memories, depending on the sensory content of those memories. Thus, the cells in dorsal areas appear to take part mainly in the retention of auditory and spatially defined memoranda. Lateral areas, on the other hand, seem to retain predominantly visual and tactile information. This apparent areal specialization in memory is deceptive, however. Whereas there are cells in those areas that respond predominantly to stimuli of one sensory modality or another, probably reflecting direct inputs from sensory areas of the posterior cortex, the majority of cells in all dorsolateral prefrontal areas seem to respond to more than one aspect of a memorandum (e.g., spatial, visual, auditory, tactile). This polysensory responsiveness is more in line with the integra- tive properties of prefrontal cells in active associative memory networks.

Prospective set. The preparatory function of the dorsolateral prefrontal cortex is teleological by definition, and just as essential as short-term memory for the temporal structuring of actions. Its role is to prepare the motor apparatus for anticipated actions and the sensory receptors for sensory signals releasing or accompanying those actions. The setting of effectors and receptors for prospective actions is the temporal mirror image of active shortterm memory. Instead of recent memory, this is "memory of the future" (Ingvar, 1985), preparing the organism for anticipated percepts and actions that will complete the bridging of a cross-temporal contingency. The underlying mechanisms are not yet known, but probably include the priming of sensory and motor structures by way of efferent connections from the dorsolateral prefrontal cortex.

In the monkey and the human, the activation of dorsolateral prefrontal cortex in prospective set is reflected by progressive increases of cell discharge and by slow surface-negative potentials before a stimulus-contingent act. The latter potentials, which are especially evident over the frontal cortex, constitute the "contingent negative variation" (CNV) and "readiness potential." Cellular activity in the dorsolateral prefrontal cortex indicates that memory and set occur by and large at the same time, both of them processed simultaneously in the period between two mutually contingent events (Quintana \& Fuster, 1999). Some prefrontal neurons engage in the memory of the cue, while others do it in the preparation of the approaching behavioral response.

Choosing one motor act among alternatives is the motor equivalent of focusing sensorial attention. Consequently, prospective set, the second cognitive function of the dorsolateral prefrontal cortex, may be properly viewed as "motor attention." It would be the focusing of attention on a motor act to secure its prompt and efficient execution. The two temporally symmetrical integrative functions of the dorsolateral prefrontal cortex, memory and set, would be paralleled by a symmetry of attentive processes supported by them. Thus, active short-term memory would be attention focused on a recent sensory event, whereas prospective set would be attention focused on an act consequent and subsequent to that event.

In the human, the pathology of prospective set adopts many forms, but most all of them can be traced to a common origin. The most characteristic disorder of prospective set, almost pathognomonic of dorsolateral frontallobe damage, is the difficulty in organizing future action, a varying degree of inability to conceptualize plans and to implement them to completion. To be sure, that deficit is often confounded by two other common symptoms of frontal pathology - namely, apathy and lack of spontaneity. These two symptoms are generally attributable to concomitant medial damage. Nonetheless, the patient 
with dorsolateral lesion, especially if large, commonly manifests a wide category of disorders of "executive system" function that can be traced not only to a deficit in the temporally retrospective function of "working memory" discussed above, but to the retrospective function of prospective set (Fuster, 1997). Those disorders include, but are not limited to, problems with "set-shifting," "conceptualization" (of the future), and "perseverative responding." The latter is essentially a regressive symptom. In the absence of perspectives in time, either backwards or forwards, the patient falls compulsively into routine and well-trodden patterns of speech or behavior.

\section{The Perception-Action Cycle}

To organize a complex new sequence of behavior, the nervous system has to perform a series of successive temporal integrations of percepts with actions. Every one of such integrations is to some extent contingent on others that have preceded it. Any motor act in the series, which is to some extent dependent on a previous percept, will induce certain changes in the environment which in turn will determine and modify subsequent perceptions, and these will in turn lead to subsequent acts. This is what I have called the "perception-action" cycle-namely, the continuous operation, toward a goal, of a cybernetic cycle of interactions of the organism with its environment. It is the neurocybernetics of a long established basic principle of biology (Uexküll, 1926).

In the course of the behavioral sequence, perceptions and acts that are mutually contingent upon one another are often separated by time, and by other intervening acts and percepts. Furthermore, the pursuit of the ultimate goal of the sequence usually requires the interim attainment of lesser goals. Consequently, the organization of the behavioral sequence necessitates the continuous mediation of contingencies across time. This is the main function of the dorsolateral prefrontal cortex in the perceptionaction cycle. This cortex is situated at the summit of the neural substrate of that cycle, which consists of two parallel hierarchies of neural structures, one sensory and the

\section{SENSORY HIERARCHY}

\section{MOTOR HIERARCHY}

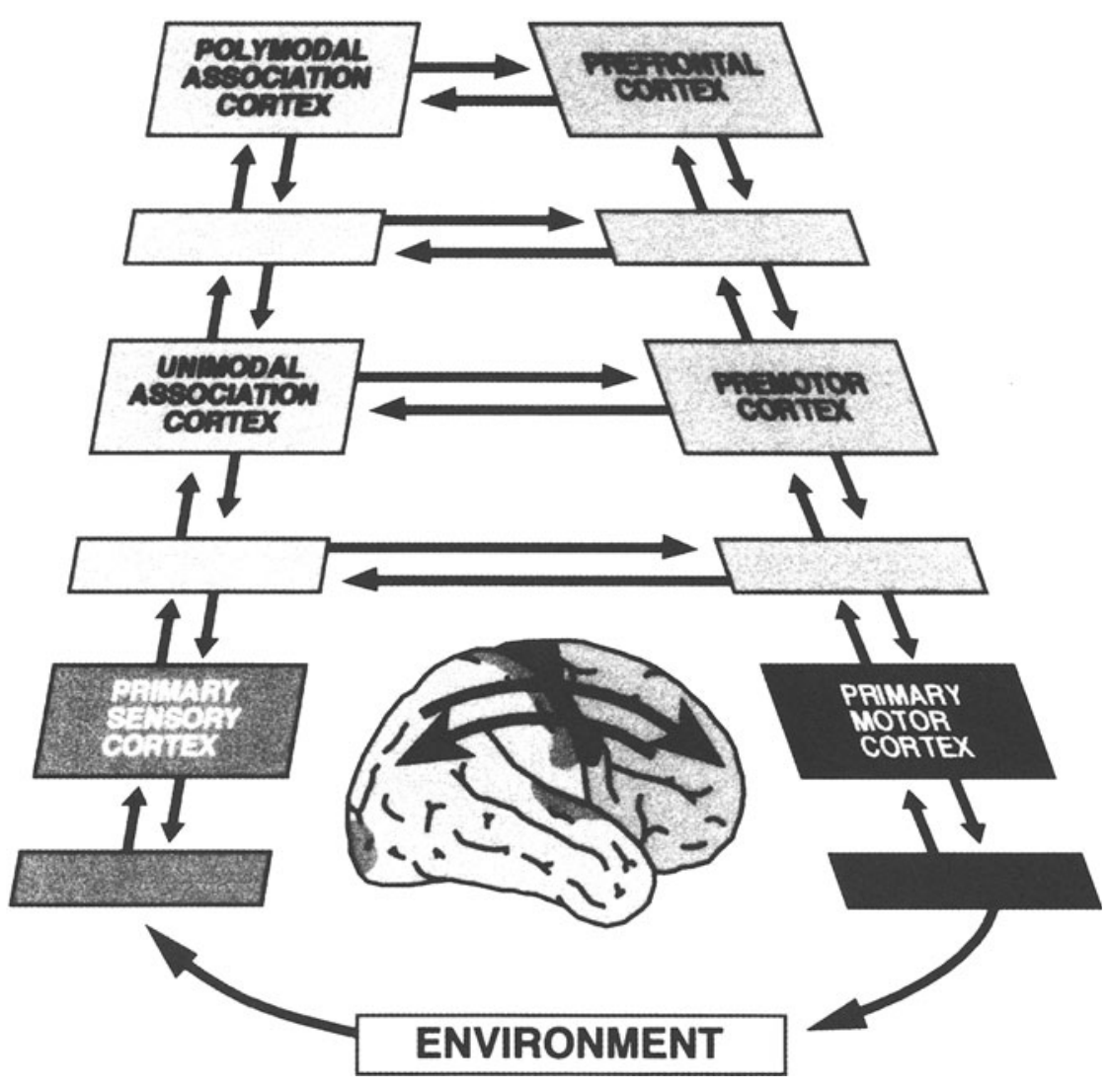

Figure 3. Cortical anatomy of the perception-action cycle depicted around a lateral view of the human brain. Unlabeled regions represent subareas of labeled regions or intermediate areas between them. All the arrows represent connections that have been demonstrated in the monkey. 
other motor, extending along the nerve axis from the spinal cord to the cerebral cortex. All stages of those hierarchies are reciprocally connected, and so is each stage with the one immediately above and the one immediately below. Figure 3 shows the cortical stages of the perceptionaction cycle.

Cross-temporal contingencies of automatic behavior, such as walking or well-rehearsed instrument playing, are mediated at subcortical stages of the neural substrate of the perception-action cycle. New and complex sequences of behavior, however, engage the cortical levels of the cycle's hierarchies. Since these behaviors usually contain numerous and complex contingencies across time, they engage regions of the neocortex, especially the dorsolateral prefrontal cortex. This cortex, in functional interaction with posterior association cortices, bridges cross-temporal contingencies at the apex of the cycle. That bridging of contingencies across time depends on the two temporal integrative functions of short-term active memory and prospective set. Their neural mechanisms are only now beginning to emerge from obscurity.

\section{REFERENCES}

AKERT, K. (1964). Comparative anatomy of frontal cortex and thalamofrontal connections. In J. M. Warren \& K. Akert (Eds.), The frontal granular cortex and behavior (pp. 372-396). New York: McGraw-Hill.

AMARAL, D. G. (1987). Memory: Anatomical organization of candidate brain regions. In F. Plum (Ed.), Handbook of physiology: Section l. The nervous system: Vol. 5. Higher functions of the brain: Pt. I (pp. 211-294). Bethesda, MD: American Physiological Society.

BaCheVAlier, J., \& MishKIN, M. (1986). Visual recognition impairment follows ventromedial but not dorsolateral prefrontal lesions in monkeys. Behavioural Brain Research, 20, 249-261.

BADDELEY, A. D. (1986). Working memory. Oxford: Oxford University Press, Clarendon Press.

BrodmanN, K. (1912). Neue Ergebnisse über die vergleichende histologische Lokalisation der Grosshirnrinde mit besonderer Berucksichtigung des Stirnhirns. Anatomische Anzeiger, 41(Suppl.), 157-216.

Cavada, C., \& Goldman-Rakic, P. S. (1989). Posterior parietal cortex in rhesus monkey: 1. Parcellation of areas based on distinctive limbic and sensory corticocortical connections. Journal of Comparative Neurology, 287, 393-421, 1989.

Cohen, J. D., Forman, S. D., Braver, T. S., Casey, B. J., ServanSChreiber, D., \& Noll, D. C. (1994). Activation of the prefrontal cortex in a nonspatial working memory task with functional MRI. Human Brain Mapping, 1, 293-304.

CONEL, J. L. (1939-1963). The postnatal development of the human cerebral cortex (6 vols.). Cambridge, MA: Harvard University Press.

Funahashi, S., Bruce, C. J., \& Goldman-Rakic, P. S. (1989). Mnemonic coding of visual space in the monkey's dorsolateral prefrontal cortex. Journal of Neurophysiology, 61, 331-349.

FUSTER, J. M. (1973). Unit activity in prefrontal cortex during delayedresponse performance: Neuronal correlates of transient memory. Journal of Neurophysiology, 36, 61-78.

Fuster. J. M. (1995). Memory in the cerebral cortex: An empirical approach to neural networks in the human and nonhuman primate. Cambridge, MA: MIT Press.

FUSTER, J. M. (1997). The prefrontal cortex: Anatomy, physiology, and neuropsychology of the frontal lobe (3rd ed.) Philadelphia: LippincottRaven.
Fuster, J. M., Bauer, R. H., \& Jervey, J. P. (1985). Functional interactions between inferotemporal and prefrontal cortex in a cognitive task. Brain Research, 330, 299-307.

Goldman-Rakic, P. S., Selemon, L. D., \& Schwartz, M. L. (1984). Dual pathways connecting the dorsolateral prefrontal cortex with the hippocampal formation and parahippocampal cortex in the rhesus monkey. Neuroscience, 12, $719-743$.

Haber, S. N., Kunishio, K., Mizobuchi, M., \& Lynd-Balta, E. (1995). The orbital and medial prefrontal circuit through the primate basal ganglia. Journal of Neuroscience, 15, 4851-4867.

HutTENLOCHER, P. R. (1979). Synaptic density in human frontal cortexdevelopmental changes and effects of aging. Brain Research, 163, 195-205.

INGVAR, D. H. (1985). "Memory of the future": An essay on the temporal organization of conscious awareness. Human Neurobiology, 4 , 127-136.

JaCobson, S., Butters, N., \& Tovsky, N. J. (1978). Afferent and efferent subcortical projections of behaviorally defined sectors of prefrontal granular cortex. Brain Research, 159, 279-296.

JERISON, H. J. (1994). Evolution of the brain. In D. W. Zaidel (Ed.), Neuropsychology (pp. 53-81). San Diego: Academic Press.

Jones, E. G., \& Powell, T. P. S. (1970). An anatomical study of converging sensory pathways within the cerebral cortex of the monkey. Brain, 93, 793-820.

Jonides, J., Smith, E. E., Koeppe, R. A., Awh, E., Minoshima, S., \& Mintun, M. A. (1993). Spatial working memory in humans as revealed by PET. Nature, 363, 623-625.

LURIA, A. R. (1966). Higher cortical functions in man. New York: Basic Books.

McCarthy, G., Blamire, A. M., Puce, A., Nobre, A. C., Bloch, G., Hyder, F., Goldman-Rakic, P., \& Shulman, R. G. (1994). Functional magnetic resonance imaging of human prefrontal cortex activation during a spatial working memory task. Proceedings of the $\mathrm{Na}$ tional Academy of Sciences, 91, 8690-8694.

Morecraft, R. J., Geula, C., \& Mesulam, M.-M. (1992). Cytoarchitecture and neural afferents of orbitofrontal cortex in the brain of the monkey. Journal of Comparative Neurology, 323, 341-358.

NikI, H. (1974). Differential activity of prefrontal units during right and left delayed response trials. Brain Research, 70, 346-349.

Pandya, D. N., \& Yeterian, E. H. (1985). Architecture and connections of cortical association areas. In A. Peters \& E. G. Jones (Eds.), Cerebral cortex (Vol. 4, pp. 3-61). New York: Plenum.

Porrino, L. J., Crane, A. M., \& Goldman-Rakic, P. S. (1981). Direct and indirect pathways from the amygdala to the frontal lobe in rhesus monkeys. Journal of Comparative Neurology, 198, 121-136.

Quintana, J., \& Fuster, J. M. (1999). From perception to action: Temporal integrative functions of prefrontal and parietal neurons. Cerebral Cortex, 9, 213-221.

Selemon, L. D., \& Goldman-Rakic, P. S. (1985). Longitudinal topography and interdigitation of corticostriatal projections in the rhesus monkey. Journal of Neuroscience, 5, 776-794.

Swartz, B. E., Halgren, E., Fuster, J. M., Simpkins, F., Gee, M., \& MANDELKERN, M. (1995). Cortical metabolic activation in humans during a visual memory task. Cerebral Cortex, 3, 205-214.

UeXKüLL, J.V. (1926). Theoretical biology. New York: Harcourt, Brace.

VAN Buren, J. M. \& Borke, R. C. (1972). Variation and connections of the human thalamus. Berlin: Springer-Verlag.

Zipser, D., Kehoe, B., Littlewort, G., \& Fuster, J. (1993). A spiking network model of short-term active memory. Journal of Neuroscience, 13, 3406-3420.

(Manuscript received August 26, 1999; revision accepted for publication April 1, 2000.) 